\title{
Procedures in Practice
}

\section{SKIN BIOPSY (2)}

\section{Choice of site}

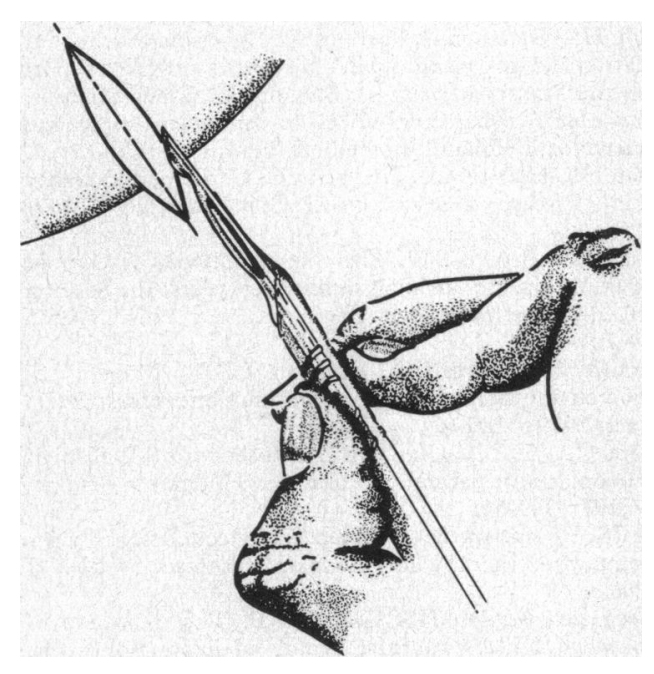

Choice of lesion-In general, a representative lesion at the height of its intensity, unmodified by trauma or treatment, will best show the histological features. The major exceptions are blisters (and pustules), which should be as new as possible and preferably less than a day old when the specimen is taken. An older blister may show confusing changes owing to regeneration, excoriation, or infection.

When the edge of the lesion is well demarcated it is usually best to take the specimen from the edge to include a small portion of normal skin. The edge is often the most active part of the lesion, and the normal skin serves as a built-in control. Sutures often hold better on normal skin. In blistering eruptions, however, perilesional skin is the best site for immunofluorescence and should form the greater proportion of the specimen. When the lesions are poorly demarcated a site of maximum activity should be sought.

Sometimes it may be necessary to take multiple specimens to assess the evolution or varied morphology of lesions.

Orientation of incision-The long axis of the wound should follow the natural wrinkle lines of the skin.

Choice of body site-Some scarring is inevitable, and the site should be chosen to minimise cosmetic disability. With keloids individual predisposition is the main factor, but the chin, midline of the chest, shoulders, and upper outer arms are areas in which the risk of keloid formation is greatest.

Sites subject to much movement, friction, or pressure are best avoided.

Healing on the lower legs is often slow but may be improved by rest and elevation or supportive bandaging.

\section{Skin tumours}

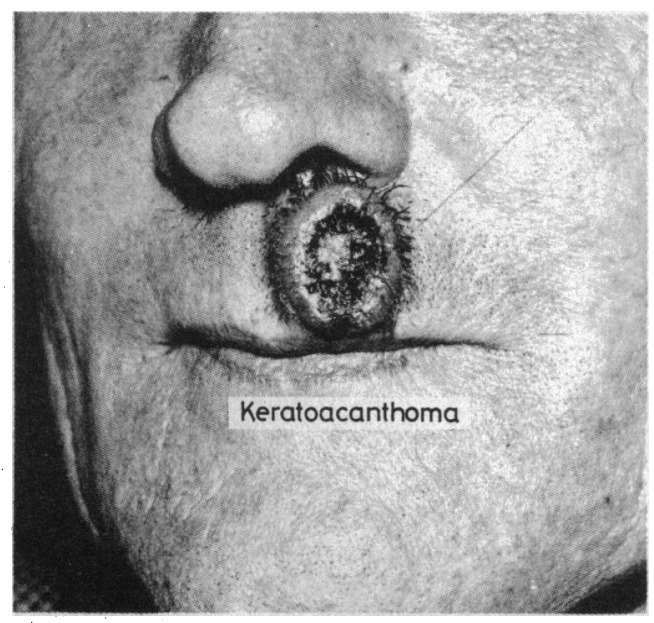

Suspected malignant melanoma-Clinical diagnosis of malignant melanoma is notoriously inaccurate. A lesion that is strongly suspected of being a melanoma should be widely excised and grafted. When a lesion might only possibly be a melanoma it is better not to inflict such an operation on the patient. The lesion should be completely excised with a minimum margin of 1-2 mm. Further surgery would of course be required should the lesion prove to be a melanoma.

Other malignant tumours-When surgical excision would be the treatment of choice for a suspected malignant tumour direct referral to the surgeon is preferable to a preliminary biopsy.

Keratoacanthoma, a benign, spontaneously regressing tumour, may be histologically confused with squamous carcinoma unless the specimen clearly shows its typical architecture. The specimen should include a segment of the shoulder of the lesion extending into the central crater, along with adjacent normal skin and subcutaneous fat. If the clinical diagnosis of keratoacanthoma can confidently be made curettage, while failing to meet all the above criteria, may be regarded as satisfactory. 


\section{Complications}

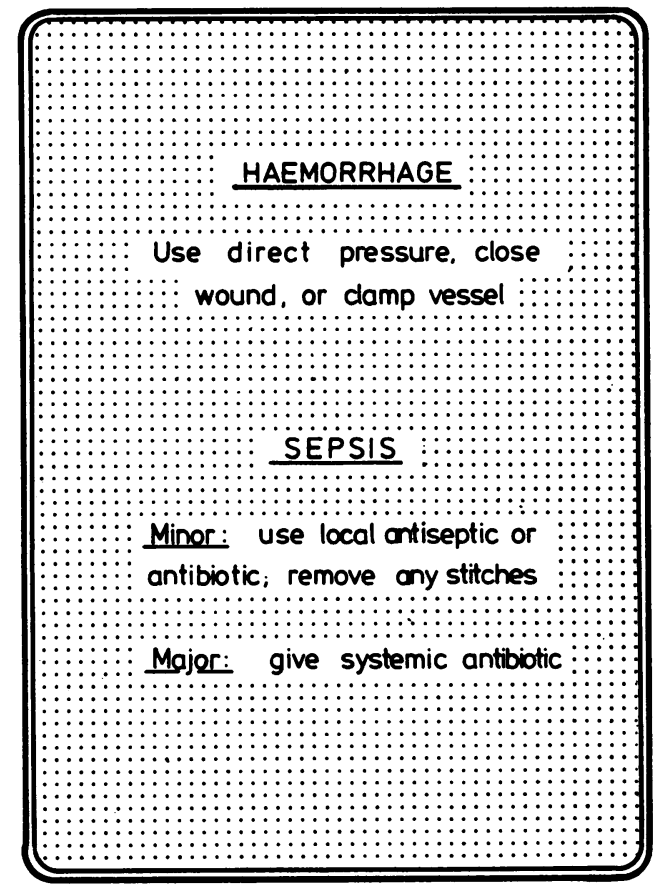

Haemorrhage-Small arteries may be cut, resulting in pulsatile bleeding. This will usually subside spontaneously or respond to direct pressure or wound closure. Occasionally the vessel may have to be clamped and a ligature of absorbable catgut applied.

Scalp incisions may bleed profusely.

Sepsis-Factors predisposing to sepsis include careless technique and occlusive dressings. Minor sepsis responds to a local antiseptic or antibiotic or to removal of stitches when appropriate. Infections with cellulitis, lymphangitis, or lymphadenitis require a systemic antibiotic.

Wounds that gape after infection seldom benefit from resuturing.

Keloids-See above.

\section{What to do with the specimen}

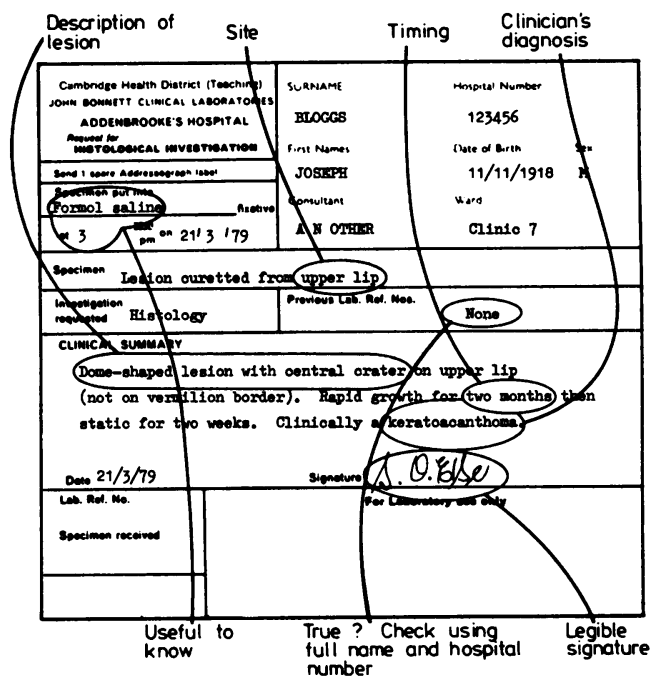

A small specimen may curl. This may be prevented by laying it flat, underside down - that is, epidermis upwards - on a small piece of blotting paper and placing the whole in the fixative. The standard fixative is formol saline.

For examination by immunofluorescence the specimen is preserved by freezing, without chemical fixative. Several techniques are used. In one a $7 \%$ gelatin solution (liquefied if necessary by warming) is poured into a small plastic capsule. The specimen is immersed directly in the gelatin and the (labelled) capsule closed and dropped into a flask containing liquid nitrogen. The specimen must remain frozen during transport to the laboratory.

When mast cells are to be examined preliminary fixation is in $70 \%$ alcohol. If a specimen has to be cultured for micro-organisms it should not be fixed at all or allowed to dry out. For other special investigationsfor example, electron microscopy-the laboratory should be consulted.

The accompanying form must contain adequate identification and a clinical summary including the suspected or differential diagnosis. A sketch of the biopsy area may be helpful. Specimens must, of course, be correctly labelled.

This is the second and concluding part of this article; the first part appeared last week

Dr Allan S Highet, BSC, MRCP, is senior registrar and Dr Robert H Champion, MB, FRCP, a consultant in the department of dermatology, Addenbrooke's Hospital, Cambridge CB2 2QQ. 